\title{
Klassische systemische Therapie der Psoriasis
}

\author{
W. K. Ludwig-Peitsch \\ N. Kemmler \\ S. Goerdt \\ M. Goebeler
}

\section{Classical Systemic Therapy of Psoriasis}

\section{Zusammenfassung}

Zur Therapie von schweren Psoriasis-Formen und der Psoriasisarthritis stehen eine Reihe klassischer Systemtherapeutika zur Verfügung, darunter Fumarsäure, Acitretin, Methotrexat und Ciclosporin. Daneben ist Leflunomid in Deutschland für die Psoriasisarthritis zugelassen; kasuistisch und im Rahmen kleinerer Studien wurde auch Mycophenolat-Mofetil erfolgreich eingesetzt. Nach Einführung der Biologicals wird zunehmend eine Neubewertung der klassischen Antipsoriatika und ihres Stellenwerts in der Psoriasistherapie gefordert. Diese Arbeit gibt einen Überblick über Einsatzmöglichkeiten, Effektivität und Sicherheitsprofil der klassischen Systemtherapeutika der Psoriasis und möglicher Kombinationen. Da die Psoriasis eine oft über mehrere Jahrzehnte chronisch verlaufende Erkrankung ist, bedarf es eines langfristigen Therapiekonzepts unter Berücksichtigung der Langzeit-Risiken und kumulativen Toxizitäten. Zur Reduktion solcher Risiken bietet sich oftmals der sequenzielle oder rotierende Einsatz der verschiedenen Systemtherapeutika an. In diesem Zusammenhang werden die Biologicals in Zukunft noch stärker als heute eine Rolle spielen; ob sie die klassischen Antipsoriatika ersetzen können werden, ist heute noch nicht absehbar.
Abstract

For therapy of severe psoriasis and psoriatic arthritis, a number of classical systemic therapeutics are available that include fumaric acid, acitretin, MTX and cyclosporine. In addition, leflunomide has been approved for the treatment of psoriatic arthritis. Moreover, casuistically and in the context of small studies, mycophenolate mofetil has been applied successfully. After the introduction of biologicals, there is an increasing demand for re-evaluating the classical systemic antipsoriatics and reappraising their value for psoriasis therapy. This article gives a survey of their efficiency, their safety profile and possible combinations. As psoriasis is a chronic disease often affecting the patients over many decades, a long-term therapy concept considering longrun risks and cumulative toxicities is required. To reduce such risks, a sequential or rotating application of the diverse systemic medications often appears suitable. In this context, biologicals will gain a greater role in the future than today, but presumably will not completely replace the classical antipsoriatics.

\section{Einleitung}

Die Psoriasis zählt mit einer Prävalenz von 2-3\% zu den häufigsten chronischen Erkrankungen und kann die Lebensqualität betroffener Patienten wesentlich einschränken [1]. Dabei korrelie- ren objektive Messkriterien zur Erfassung des Schweregrads wie der PASI-Score oft nur unzureichend mit den subjektiven Auswirkungen auf die Lebensqualität. Die meisten Patienten leiden unter leichten bis mittelschweren Psoriasisformen, die mit topischen Therapien wie Salicylsäure, Dithranol, Vitamin D- und Vi- 
tamin A-Analoga sowie topischen Glukokortikoiden bzw. UVTherapien zufriedenstellend kontrolliert werden können. Darüber hinaus lassen sich häufig auch schwerere Formen effektiv mit Dithranol in Kombination mit einer Lichttherapie behandeln. Für einen Teil der Patienten reichen diese Therapieoptionen allerdings nicht aus. Hierzu zählen Patienten mit ausgedehnter Plaque-Psoriasis, die auf die genannten Maßnahmen refraktär ist, und Patienten mit erythrodermatischen und pustulösen Formen der Psoriasis. Andererseits kommen auch Patienten mit einer lokalisierten, aber therapierefraktären und körperlich behindernden Psoriasis, z. B. einer Psoriasis palmoplantaris, für eine systemische Therapie in Frage. Gleiches gilt für Patienten mit einer mittelschweren Psoriasis, bei denen eine Lichttherapie kontraindiziert ist, z. B. aufgrund maligner Hauttumoren in der Anamnese (Übersicht: [2]). Schließlich wird bei einer aktiven Psoriasisarthritis mit der Gefahr einer Gelenkdestruktion fast immer eine systemische Therapie erforderlich sein.

Hierfür stehen einerseits die klassischen Psoriasistherapeutika zur Verfügung. In Deutschland zugelassen sind Fumarsäureester, Acitretin, Methotrexat und Ciclosporin; Leflunomid besitzt eine Zulassung für die Indikation Psoriasisarthritis. Darüber hinaus kommt gelegentlich das bisher nicht zugelassene Immunsuppressivum Mycophenolat-Mofetil zum Einsatz. Andererseits wurden in den letzten Jahren Biologicals wie Etanercept, Infliximab und Efalizumab in die Psoriasistherapie eingeführt und haben das Therapiespektrum wesentlich bereichert. In diesem $\mathrm{Zu}$ sammenhang kommt es zunehmend zu Diskussionen über die Rolle, die Effizienz und das Sicherheitsprofil der klassischen Systemtherapeutika, und es wird Aufgabe der nächsten Jahre sein, ihren Stellenwert im Zeitalter der Biologicals zu definieren.

\section{Fumarsäureester}

Erstmals wurde 1959 durch den deutschen Chemiker Schweckendieck über einen erfolgreichen Einsatz von Fumarsäureestern in der Psoriasistherapie berichtet. Schweckendieck, der selbst unter einer Psoriasis litt, testete verschiedene Zubereitungen von Fumarsäure und ihren Estern im Selbstversuch [3]. Seither werden Fumarsäureester v. a. im deutschen Sprachraum und in den Niederlanden eingesetzt. Eine definierte Mischung von Fumarsäureestern, Fumaderm ${ }^{\circledR}$, ist in Deutschland zur Psoriasistherapie zugelassen und ist hier das am häufigsten verordnete systemische Antipsoriatikum. Fumarsäureester werden aufgrund ihres Nebenwirkungsprofils nach einem vom Hersteller vorgegebenen Schema über mehrere Wochen einschleichend dosiert $[4,5]$ : Während der ersten Behandlungswoche erhalten die Patienten zunächst eine Tablette einer niedrig dosierten Formulation, Fumaderm ${ }^{\circledR}$ initial (30 mg Dimethylfumarat und $75 \mathrm{mg}$ Monoethylfumarat), die während der nächsten acht Wochen bis zur Maximaldosis von $3 \times 2$ Tbl. des höher dosierten Präparats, Fumaderm ${ }^{\circledR}$ (120 mg Dimethylfumarat und 95 mg Monoethylfumarat), gesteigert wird.

\section{Wirkmechanismus}

Fumarate bewirken eine Hemmung der T-Zell-Funktion und führen zu einem Shift von der bei der Psoriasis vorherrschenden Th1-Immunantwort in Richtung eines Th2-Reaktionsmusters. Fumarate inhibieren weiterhin den Transkriptionsfaktor NF- $\mathrm{B}$, was u. a. eine Hemmung der Expression proinflammatorischer Zytokine und Adhäsionsmoleküle zur Folge hat.

\section{Effektivität}

Während nach den Erstberichten von Schweckendiek zunächst nur Fallberichte und kleinere Fallserien publiziert worden waren, wurde 1990 erstmals eine randomisierte, doppelblinde Studie zum Einsatz von Fumarsäureestern vorgelegt [6]. Diese zeigte in der Patientengruppe, die mit einer Mischung aus Monoethylfumarat und Dimethylfumarat behandelt wurde, nach 16 Wochen eine Reduktion der befallenen Körperoberfläche von initial durchschnittlich $21 \%$ auf 6,7\%. Eine solche Kombination aus Monoethylfumarat und Dimethylfumarat wurde später als Handelspräparat Fumaderm ${ }^{\circledR}$ eingeführt. Von Altmeyer und Kollegen stammt eine randomisierte, doppelblinde und Plazebo-kontrollierte Studie, in der nach 4 Monaten Therapiedauer mit Fumaderm $^{\circledR}$ bei $71 \%$ der Patienten ein Ansprechen und eine mittlere PASI-Reduktion um 50\% gefunden wurde [7]. In einer prospektiven Multizenter-Studie fanden Mrowietz et al. sogar eine durchschnittlich $80 \%$ ige Senkung des PASI-Scores [8].

Gemäß den aktuellen Richtlinien wird empfohlen, Fumarsäureester mit topischen Therapien zu kombinieren [5]. Nach einer randomisierten, doppelblinden Studie an 143 Patienten, in der Fumaderm plus topisch appliziertes Calcipotriol mit einer Fumaderm-Monotherapie verglichen wurde, konnte eine durchschnittlich 50\%ige PASI-Reduktion in der ersten Gruppe bereits nach 3 Wochen erzielt werden, bei Monotherapie mit Fumaderm $^{\circledR}$ erst nach 9 Wochen [9].

\section{Sicherheitsprofil}

Die häufigsten, bei mehr als $2 / 3$ der Patienten beobachteten unerwünschten Wirkungen betreffen den Gastrointestinaltrakt (Tab. 1; [5,10]). Dabei können die Beschwerden von mildem Unwohlsein bis zu schweren Magen-Darmkrämpfen reichen. Sie setzen meist 4-12 Wochen nach Therapiebeginn ein und können später rückläufig sein, erfordern aber recht häufig eine Dosisreduktion. Darüber hinaus kommt es bei etwa 1/3 der Patienten zu einer unterschiedlich stark ausgeprägten Flush-Symptomatik. Diese Nebenwirkungen sind zwar ungefährlich, werden aber subjektiv als sehr störend empfunden und führen - zusammen mit unzureichender Wirksamkeit - zu einer recht hohen Rate von Therapieabbrüchen, die zwischen 30 und 40\% liegt [8].

Eine weitere Nebenwirkung, die unbedingt kontrolliert werden sollte, ist ein Abfall der Lymphozytenzahl. Diese wird bei nahezu allen mit Fumarsäureestern behandelten Patienten beobachtet [10] und führt bei ca. einem Zehntel der Patienten zu Werten von unterhalb 50\% des Ausgangsbefundes. Bis heute wurde in diesem Zusammenhang kein Anhalt für eine erhöhte Inzidenz von Infekten gefunden. Dennoch ist bei einem Abfall der Leukozytenzahlen unter 3,0 $\times 10^{9} / 1$ oder der Lymphozyten unter $0,5 \times 10^{9} / 1$ eine Dosisreduktion vorzunehmen. Bei etwa der Hälfte der Patienten kann es außerdem zu einer transienten Eosinophilie kommen. Aus diesen Gründen ist Fumarsäure bei Patienten mit vorbestehenden hämatologischen Erkrankungen kontraindiziert.

In älteren Fallberichten wurde im zeitlichen Zusammenhang mit der Einnahme von Fumarsäureestern ein akutes Nierenversagen dokumentiert. Diese Toxizität konnte in größeren Studien nicht 
Tab. 1 Kontraindikationen und Nebenwirkungen der klassischen systemischen Antipsoriatika

\begin{tabular}{|c|c|c|}
\hline Wirkstoff & Kontraindikationen & Nebenwirkungen \\
\hline Fumarsäure & $\begin{array}{l}\text { Gastrointestinale Ulzera, schwere Leber- oder Nierenfunktions- } \\
\text { störungen, hämatologische Erkrankungen, Überempfindlichkeit } \\
\text { gegen Fumarsäureester } \\
\text { Schwangerschaft und Stillzeit (fehlende Erfahrung) }\end{array}$ & $\begin{array}{l}\text { Häufig: Diarrhoe, Völlegefühl, krampfartige Oberbauchschmerzen, } \\
\text { Flush-Symptomatik, Leukopenie, Lymphopenie, Eosinophilie } \\
\text { Selten: Erhöhung des Kreatinin, Proteinurie, Leberwerterhöhungen, } \\
\text { unspezifische Knochenschmerzen }\end{array}$ \\
\hline Acitretin & $\begin{array}{l}\text { Leberfunktionsstörungen, Alkoholabusus, vorbestehende Fettstoff- } \\
\text { wechselstörungen, manifester Diabetes mellitus, Niereninsuffizienz, } \\
\text { Tragen von Kontaktlinsen, Überempfindlichkeit gegen Acitretin } \\
\text { Schwangerschaft und Stillzeit (absolut), Kinderwunsch in den } \\
\text { nächsten } 2 \text { Jahren (nur für Frauen; für Männer keine Einschränkung) }\end{array}$ & $\begin{array}{l}\text { Häufig: Mukokutane Xerose, Rhinitis sicca, Konjunctivitis sicca, } \\
\text { Nachtblindheit, Haarausfall, Hepatotoxizität, gastrointestinale } \\
\text { Beschwerden, Anstieg der Triglyceride } \\
\text { Selten: Verschlechterung eines Diabetes, Muskel- und Gelenk- } \\
\text { schmerzen, Hyperostosen, Sehnen- und Ligament-Kalzifikationen } \\
\text { Teratogenität (Retinoid-Embryopathie) }\end{array}$ \\
\hline Methotrexat & $\begin{array}{l}\text { Leberfunktionsstörungen, Alkoholabusus, Niereninsuffizienz, } \\
\text { Immundefizienz, hämatologische Erkrankungen, aktive Infektionen, } \\
\text { gastrointestinale Ulzera, Stomatitis, Überempfindlichkeit gegen } \\
\text { Methotrexat, gleichzeitige Lichttherapie } \\
\text { Schwangerschaft und Stillzeit (absolut), Kinderwunsch (für Frauen } \\
\text { und Männer Konzeption frühestens } 3 \text { Monate nach Therapieende) }\end{array}$ & $\begin{array}{l}\text { Häufig: Übelkeit, Erbrechen, Stomatitis, Haarausfall, verstärkte } \\
\text { Infektanfälligkeit, Anstieg der Transaminasen } \\
\text { Selten: Leberfibrose oder -zirrhose, Myelosuppression, Exantheme, } \\
\text { Vaskulitiden, erhöhte Photosensitivität, Pneumonitis, Verschlech- } \\
\text { terung der Nierenfunktion, erhöhtes Malignomrisiko } \\
\text { Teratogenität }\end{array}$ \\
\hline Ciclosporin & $\begin{array}{l}\text { Niereninsuffizienz, unkontrollierte arterielle Hypertonie, Malignome } \\
\text { (auch anamnestisch), Immundefizienz, floride oder chronische } \\
\text { Infekte, schwere Leberfunktionsstörung, Überempfindlichkeit gegen } \\
\text { Ciclosporin, gleichzeitige Lichttherapie } \\
\text { Schwangerschaft (relativ), Stillzeit (absolut) }\end{array}$ & $\begin{array}{l}\text { Häufig: Niereninsuffizienz, Blutdruckanstieg, Anstieg der Leberwerte, } \\
\text { gastrointestinale Beschwerden, Hypertrichose, Gingivahyperplasie, } \\
\text { Tremor, Müdigkeit, Parästhesien } \\
\text { Selten: allergisches Exanthem, Pankreatitis, opportunistische } \\
\text { Infektionen, Anämie, Thrombopenie, erhöhtes Malignomrisiko }\end{array}$ \\
\hline Leflunomid & $\begin{array}{l}\text { Zulassung in Deutschland nur zur Behandlung der } \\
\text { Psoriasisarthritis } \\
\text { Lebererkrankungen, Niereninsuffizienz, Immundefekt, schwere } \\
\text { Infektion, hämatologische Erkrankungen, Überempfindlichkeit gegen } \\
\text { Leflunomid } \\
\text { Schwangerschaft und Stillzeit (absolut), Kinderwunsch (für Frauen } \\
\text { keine Konzeption bis } 2 \text { Jahre nach Therapieende, für Männer bis } \\
3 \text { Monate) }\end{array}$ & $\begin{array}{l}\text { Häufig: gastrointestinale Beschwerden, Leberwerterhöhungen, } \\
\text { Blutdruckanstieg, Haarausfall, Pruritus, leichte allergische Reaktionen, } \\
\text { Schwindel, Sehnenscheidenentzündung, Stomatitis, Infektionen } \\
\text { Selten: schwere allergische Reaktion, Blutbildveränderungen, } \\
\text { schwere Leberschädigung, Sehnenruptur, interstitielle Pneumonie, } \\
\text { erhöhtes Malignomrisiko } \\
\text { Teratogenität }\end{array}$ \\
\hline
\end{tabular}

bestätigt werden (Mrowietz et al., 1999; Ormerod und Mrowietz, 2004). Nichtsdestotrotz sollte Fumarsäure nicht bei Patienten mit Nierenfunktionsstörungen angewendet werden. Im ersten halben Jahr der Therapie werden monatliche, danach zweimonatliche Kontrollen der Nieren- und Leberwerte sowie des Differenzialblutbildes empfohlen (Tab. 2).

Nach den aktuellen Richtlinien ist eine kurzfristige intermittierende Therapie oder alternativ eine kontinuierliche Therapie für maximal zwei Jahre angezeigt [5]. Es existieren aber auch Hinweise, dass über einen weit längeren Zeitraum eine sichere Therapie möglich ist.

\section{Zusammenfassende Beurteilung}

Erfahrungen in Deutschland und Nordeuropa belegen, dass Fumarsäureester effektiv und relativ sicher zur Behandlung mittelschwerer und schwerer Psoriasisformen eingesetzt werden können. Randomisierte Studien, in denen Fumarsäureestern anderen systemischen Antipsoriatika gegenübergestellt werden, fehlen bisher, was einen Effektivitätsvergleich erschwert. Nach klinischen Erfahrungen erscheinen die Fumarate allerdings weniger stark wirksam zu sein als MTX oder Ciclosporin. Unter Therapie treten unerwünschte Wirkungen wie gastrointestinale Beschwerden und Flush-Symptomatik mit großer Häufigkeit auf. Diese sind zwar meist harmlos und nach dem Absetzen schnell reversibel, werden aber von den Patienten häufig als sehr unangenehm empfunden und resultieren in einer nicht unerheblichen Therapieabbruchrate. Ein Fumurat der 2.Generation, BG00012 (Dimethylfumarat), wird gegenwärtig in klinischen
Studien evaluiert und scheint weniger gastrointestinale Symptome hervorzurufen.

\section{Retinoide}

Das erste systemische Retinoid, das seit den späten 1970er Jahren erfolgreich zur Psoriasistherapie eingesetzt wurde, war Etretinat. Dieses Präparat ist stark lipophil und akkumuliert im Fettgewebe, von wo es erst mit einer Halbwertszeit von 120 Tagen eliminiert wird. Sein aktiver Metabolit, Acitretin, ist 50-fach weniger lipophil und besitzt mit 50 h eine wesentlich kürzere Eliminations-Halbwertszeit und damit ein weit günstigeres pharmakokinetisches Profil. Acitretin kommt Etretinat hinsichtlich seiner Effektivität gleich und hat dieses in den 1990er Jahren abgelöst. Zu Beginn einer Acitretin-Therapie sind Dosierungen zwischen 0,3 und $0,5 \mathrm{mg} / \mathrm{kg} \mathrm{KG} / \mathrm{Tag}$ empfohlen, zur Erhaltungstherapie reicht manchmal auch eine geringere Dosis aus, die individuell angepasst werden kann [11].

\section{Wirkmechanismus}

Ähnlich wie Steroidhormone passieren Retinoide die Zellmembran, werden in den Zellkern transportiert und binden dort an Retinoidrezeptoren, die mit DNA interagieren und die Transkription ihrer Zielgene regulieren. Dabei existieren zwei Familien von Retinoidrezeptoren, Retinolsäurerezeptoren („retinoic acid receptors“, RAR) und Retinoid X-Rezeptoren (RXR), die jeweils in drei Subtypen, $\alpha, \beta$ und $\gamma$, vorkommen. Etretinat und Acitretin binden an RAR, und zwar an alle drei Subtypen, und aktivieren 
Tab. 2 Monitoring unter klassischer antipsoriatischer Systemtherapie

\begin{tabular}{|c|c|c|}
\hline Wirkstoff & Monitoring vor Therapiebeginn & Monitoring unter Therapie \\
\hline Fumarsäureester & Großes Blutbild, Leber- und Nierenwerte, Urinsediment & $\begin{array}{l}\text { In den ersten } 3 \text { Monaten alle } 2 \text { Wochen, danach alle } 4 \text { Wochen: } \\
\text { großes Blutbild, Leber- und Nierenwerte, Urinsediment }\end{array}$ \\
\hline Acitretin & $\begin{array}{l}\text { Kleines Blutbild, Leberwerte, Triglyceride, Gesamt-Cholesterin, } \\
\text { Kreatinin, Glukose, bei gebärfähigen Frauen Schwangerschaftstest } \\
\text { zweimal im Abstand von } 4 \text { Wochen }\end{array}$ & $\begin{array}{l}\text { Nach } 4 \text { Wochen, danach alle } 3 \text { Monate: } \\
\text { Leberwerte, Triglyceride, Gesamt-Cholesterin, } \\
\text { bei gebärfähigen Frauen alle } 28 \text { Tage Schwangerschaftstest während } \\
\text { der Therapie und danach (siehe Text), } \\
\text { bei Langzeittherapie Röntgen der Wirbelsäule, des Handskeletts und } \\
\text { der langen Röhrenknochen }\end{array}$ \\
\hline Methotrexat & $\begin{array}{l}\text { Großes Blutbild, Leber- und Nierenwerte, ggf. Hepatitisserologie, } \\
\text { bei gebärfähigen Frauen Schwangerschaftstest } \\
\text { Röntgen-Thorax }\end{array}$ & $\begin{array}{l}\text { Im 1. Monat wöchentlich, im 2. und 3. Monat zweiwöchentlich, } \\
\text { danach alle } 4 \text { Wochen: } \\
\text { großes Blutbild, Leberwerte, Kreatinin, } \\
\text { nach einer kumulativen Dosis von 1,5 g MTX Indikation zur Durch- } \\
\text { führung einer Leberbiopsie prüfen, alternativ Bestimmung des PIIINP }\end{array}$ \\
\hline Ciclosporin & $\begin{array}{l}\text { Großes Blutbild, Leber- und Nierenwerte, Elektrolyte, Urinstatus, } \\
\text { Blutdruckmessung } \\
\text { Röntgen-Thorax }\end{array}$ & $\begin{array}{l}\text { In den ersten } 2 \text { Monaten alle } 1 \text { - } 2 \text { Wochen, danach alle } 4 \text { Wochen: } \\
\text { kleines Blutbild, Leberwerte, Kreatinin, Kalium, Urinstatus, Blut- } \\
\text { druckmessung, } \\
\text { halbjährlich Kreatinin-Clearance, bei langfristiger Therapie halb- } \\
\text { jährliche Untersuchung auf Hauttumoren }\end{array}$ \\
\hline Leflunomid & $\begin{array}{l}\text { Großes Blutbild, Leber- und Nierenwerte, bei gebärfähigen Frauen } \\
\text { Schwangerschaftstest, Blutdruckmessung }\end{array}$ & $\begin{array}{l}\text { In den ersten } 6 \text { Monaten alle } 2 \text { Wochen, danach alle } 8 \text { Wochen: } \\
\text { großes Blutbild, Leberwerte, Blutdruckmessung }\end{array}$ \\
\hline
\end{tabular}

diese, während ein anderes Retinoid, Tazaroten, spezifisch mit RAR- $\beta$ und RAR- $\gamma$ interagiert [12]. Retinoide regulieren Differenzierung und Proliferation von Epithelzellen, wobei der genaue Wirkmechanismus bisher nicht bekannt ist. Histologisch ließen sich bei der Psoriasis nach Acitretin-Gabe eine Verdünnung des Stratum corneum und eine Abnahme der epidermalen und dermalen Entzündung nachweisen.

\section{Klinische Erfahrungen}

Die Wirksamkeit von Acitretin wurde in den 1980er Jahren in Plazebo-kontrollierten Studien umfassend untersucht (Übersichten: $[13,14])$. Als Monotherapie für die chronische PlaqueTyp Psoriasis ist Acitretin zwar wirksam, zeigt jedoch ein insgesamt eher mäßiges Ansprechen [13]. Hingegen ist es bei pustulösen und erythrodermatischen Formen der Psoriasis deutlich effektiver. Nach klassischen Empfehlungen sollte bei pustulöser Psoriasis eine höhere Retinoid-Dosis - bis zu 0,75 mg/kg KG/Tag - eingesetzt werden, wohingegen bei der Psoriasiserythrodermie niedrigere Dosierungen ausreichen. Diese Vorgaben stimmen allerdings nicht immer mit praktischen Erfahrungen überein: So spricht ein Teil der Patienten mit pustulöser Psoriasis bereits gut auf niedrige Retinoid-Dosierungen an, während manche Patienten mit einer Erythrodermie auf eine höhere Dosis angewiesen sind.

Wirksamkeitsvergleiche von Retinoiden (Etretinat) und Ciclosporin bei schwerer Psoriasis fielen insgesamt zugunsten von Ciclosporin aus, wobei allerdings hauptsächlich Patienten mit einer Psoriasis vom Plaque-Typ untersucht wurden (z. B. [15]). Prinzipiell eignet sich Acitretin sowohl zur Induktion einer Remission als auch als längerfristige Erhaltungstherapie im Rahmen eines sequentiellen Therapieregimes. So wird z. T. empfohlen, bei schwerer Psoriasis zunächst mit Ciclosporin eine Remission zu induzieren und anschließend als Erhaltungstherapie auf Acitretin umzustellen [16].
Retinoide lassen sich hervorragend mit einer Lichttherapie kombinieren. Vorteile einer solchen Kombination sind einerseits ein schnelleres Ansprechen, andererseits aber auch eine Reduktion unerwünschter Ereignisse durch Reduktion der kumulativen UV- und Retinoiddosen. Vielfach praktiziert und in der Wirksamkeit gut belegt sind dabei Kombinationen von Acitretin mit PUVA („Re-PUVA“, z. B. [17]) und mit UVB [18]. Dabei hat sich in letzter Zeit Schmalspektrum-UVB $(311 \mathrm{~nm})$ als besonders effektiv erwiesen [19]. Außerdem erscheinen Kombinationen mit topischen Therapien, z. B. mit Vitamin D-Analoga, sinnvoll: In einer größeren Plazebo-kontrollierten Studie ließ sich mit Acitretin plus Calcipotriol bei $67 \%$ der Patienten eine wesentliche Verbesserung erzielen, gegenüber $41 \%$ bei einer Monotherapie mit Acitretin [20].

\section{Sicherheitsbedenken}

Ein entscheidendes Risiko der Retinoide ist ihre Teratogenität. Im ersten Trimenon der Schwangerschaft können sie eine RetinoidEmbryopathie hervorrufen, die mit Neuralrohrdefekten sowie mit okulären, auditorischen, zentralnervösen und kardiovaskulären Defekten einhergehen kann. Daher sind Retinoide in der Schwangerschaft und Stillzeit streng kontraindiziert. Frauen im fortpflanzungsfähigen Alter sind von Retinoid-Therapien ausgeschlossen, es sei denn, dass gleichzeitig zwei wirksame Verhütungsmethoden angewendet werden. In Deutschland ist eine sichere Kontrazeption bis zwei Jahre nach Ende der Retinoid-Therapie vorgeschrieben [11], in anderen Ländern, z. B. in den USA, sogar bis drei Jahre nach Therapieende. Diesen Richtlinien liegt zugrunde, dass Acitretin - entweder spontan bzw. insbesondere bei zusätzlicher Ingestion von Alkohol - zu Etretinat abgebaut werden kann, welches erst nach drei Jahren zu 98\% aus dem Körper eliminiert ist. 
Weitere unerwünschte Wirkungen betreffen die Leber und die Blutfettwerte; demzufolge sind Retinoide auch bei Patienten mit bekannten Leberfunktionsstörungen und Fettstoffwechselstörungen kontraindiziert. Leberwert-Erhöhungen, die in der Regel nach dem Absetzen der Therapie reversibel sind, werden bei ca. 1/3 der Patienten beobachtet; sehr selten treten schwerwiegende Leberschädigungen auf. Die Triglyceride können unter Therapie um $60 \%$ oder mehr ansteigen; zusätzlich treten bei etwa einem Drittel der Patienten Hypercholesterinämien auf [11]. Diese sollten zunächst mittels diätetischer Maßnahmen und Dosisreduktionen angegangen werden. Darüber hinaus kann auch der Einsatz von Fibraten hilfreich sein.

Regelmäßig und fast unumgänglich kommt es zu einer mukokutanen Xerose, insbesondere zu einer Cheilitis, manchmal auch zu einer Konjunktivitis sicca, denen mit rückfettender Pflege und ggf. mit Tränenersatzflüssigkeit entgegengewirkt werden kann. Zusätzlich wird bei bis zu 75\% der Patienten ein dosisabhängig auftretender, nach Therapieende reversibler Haarausfall beobachtet. Seltenere Nebenwirkungen schließen Myalgien, Arthralgien sowie Knochenveränderungen, einschließlich vorzeitigem Epiphysenschluss, Hyperostosen und Kalzifikationen von Sehnen und Bändern, ein. Die meisten unerwünschten Wirkungen von Retinoiden können jedoch mit einfachen Mitteln durch Anamnese, klinische Untersuchung und Standard-Laboruntersuchungen erfasst werden und führen nicht zu schwerwiegenden Schäden. Insgesamt brechen nur etwa 10-20\% der mit Acitretin behandelten Patienten die Therapie aufgrund von Nebenwirkungen vorzeitig ab.

\section{Zusammenfassende Beurteilung}

Orale Retinoide sind als Monotherapie weniger effektiv als Ciclosporin oder MTX. Sie lassen sich aber ausgezeichnet mit einer Lichttherapie kombinieren und eignen sich darüber hinaus als Erhaltungstherapie. Insgesamt ist ihre Anwendung - gerade im Vergleich mit MTX und Ciclosporin - relativ sicher, und kumulative Toxizitäten existieren fast nicht. Darüber hinaus sind Retinoide neben Fumarsäureestern eines der wenigen systemischen Antipsoriatika, die selbst nicht immunsuppressiv wirken und somit für immunsupprimierte Patienten in Frage kommen. Für solche Patienten können sie sogar tumorprotektiv wirken [21]. Die Anwendung von Retinoiden wird durch ihre Teratogenität limitiert. So sollte Acitretin bei Frauen in gebärfähigem Alter nur im Ausnahmefall und nur dann verordnet werden, wenn eine absolut sichere Kontrazeption gewährleistet und begleitender Alkoholkonsum ausgeschlossen ist. Bezüglich Tazaroten, welches seit 1997 als topisches Antipsoriatikum zugelassen ist, laufen derzeit ermutigende Phase-III-Studien zur systemischen Anwendung [14].

\section{Methotrexat}

Nachdem 1951 entdeckt worden war, dass Aminopterin, ein Derivat des Methotrexat (MTX), zum Abheilen psoriatischer Hautveränderungen führen kann, wurde MTX in den 1960er Jahren als erste antipsoriatische Systemtherapie in den klinischen Alltag eingeführt. Es ist sowohl bei schweren akuten Psoriasisformen wie pustulöser oder erythrodermatischer Psoriasis als auch bei chronisch-stationärer Plaque-Psoriasis effektiv [22] und hat außerdem einen besonders hohen Stellenwert in der Therapie der Psoriasisarthritis.

Zur Therapie der Psoriasis und der Psoriasisarthritis wird MTX einmal wöchentlich in Dosierungen zwischen 7,5 und 25 mg verabreicht, wobei sowohl eine orale Einnahme als auch subkutane, intravenöse oder intramuskuläre Injektionen möglich sind. Manche Autoren empfehlen, mit einer eher niedrigen Dosis zwischen 7,5 und $15 \mathrm{mg} z \mathrm{u}$ beginnen und alle zwei bis vier Wochen um 2,5 mg zu steigern, bis eine zufriedenstellende Wirkung eintritt. Die Gabe kann entweder als Einmaldosis oder nach dem Weinstein-Schema verteilt auf drei Einzeldosen im Abstand von $12 \mathrm{~h}$ erfolgen [23] und sollte immer mit Folsäure kombiniert werden (siehe unten).

\section{Wirkmechanismus}

MTX ist ein synthetischer Folsäureantagonist, der kompetitiv das Enzym Dihydrofolat-Reduktase hemmt und so die Reduktion von Folsäure zu Tetrahydrofolat verhindert. Tetrahydrofolat wirkt als Koenzym für den Transfer von Kohlenstoff-Einheiten, die für die Biosynthese von Purinbasen und damit für den Aufbau von Nukleinsäuren erforderlich sind. Somit inhibiert MTX die DNS-Synthese und bewirkt einen Arrest des Zellzyklus in der S-Phase. Besonders betroffen sind hiervon Zellen, die sich schnell teilen, z. B. T-Lymphozyten. Zusätzlich unterdrückt es die Sekretion verschiedener Zytokine, wie Interleukin-1, Interferon- $\gamma$ und TNF- $\alpha$, und wirkt antiproliferativ auf Keratinozyten, allerdings erst in 100fach höherer Konzentration als auf Lymphozyten. Es greift somit auf verschiedenen Ebenen in die Pathogenese der Psoriasis ein.

\section{Klinische Erfahrungen}

MTX ist weltweit das am meisten eingesetzte antipsoriatische Systemtherapeutikum. Wie bei vielen Therapieansätzen, die vor dem Zeitalter der evidenzbasierten Medizin etabliert wurden, beruht sein Einsatz auf positiven Erfahrungsberichten und stützt sich auf kleinere Fallserien; kontrollierte Studien hingegen gibt es nur wenige [13]. Bei Untersuchungen zur Dosisfindung in den 1960er und 70er Jahren zeigte sich bei wöchentlicher Gabe von 25 mg MTX eine komplette Abheilung bei 50\% und eine $75 \%$ ige Besserung bei $90 \%$ der Patienten innerhalb von vier Wochen [24]. Eine Interpretation dieser Daten und ein Vergleich mit neueren Studien wird allerdings dadurch erschwert, dass quantifizierbare Erfolgskriterien wie der PASI-Score damals noch nicht eingeführt waren. Aus jüngerer Zeit stammt eine randomisierte Studie zum Vergleich von MTX mit Ciclosporin [25]. Hierin erreichten in der mit MTX behandelten Patientengruppe 64\% nach 16 Wochen den PASI-75. Allerdings mussten 23\% der Patienten die MTX-Therapie aufgrund einer Hepatotoxizität abbrechen. Im direkten Vergleich erwiesen sich MTX und Ciclosporin A als ähnlich effektiv. Eine in Indien durchgeführte Vergleichsstudie von MTX versus Ciclosporin ergab ein schnelleres Ansprechen von MTX: Hier besserte sich der PASI-Score bereits nach einem Monat Behandlungsdauer um 70\%, die verabreichte MTXDosis lag mit durchschnittlich $27,7 \mathrm{mg} /$ Woche allerdings auch etwas höher [26].

Zur Therapie einer Psoriasisarthritis, die auf nichtsteroidale Antiphlogistika nicht anspricht, gilt MTX als ein Therapeutikum der ersten Wahl. Für eine detaillierte Übersicht zu diesem Indika- 
tionsgebiet sei auf den Artikel von Kneitz et al. in dieser Ausgabe der Aktuellen Dermatologie verwiesen.

\section{Sicherheitsbedenken}

MTX kann zu einer Reihe schwerwiegender Toxizitäten führen, weswegen unter Therapie eine sorgfältige Überwachung der Patienten erforderlich ist. In erster Linie können Knochenmark, Leber, Niere und Lunge betroffen sein, wobei das Toxizitätsrisiko bei einer Nierenfunktionsstörung stark erhöht ist. Demzufolge ist MTX auch bei Patienten mit hämatologischen Erkrankungen, chronischen Infektionen, Lebererkrankungen, Alkoholabusus und Nierenfunktionsstörungen kontraindiziert (vgl. Tab.1).

Eine entscheidende Nebenwirkung von MTX ist seine Hepatotoxizität mit der möglichen Folge einer Leberfibrose oder -zirrhose. Die Häufigkeit, mit der beides auftritt, wird überaus kontrovers diskutiert [27]: In früheren Studien wurden Inzidenzen von bis zu 26\% angegeben. Heute sind diese Zahlen, nicht zuletzt als Folge sorgfältiger Patientenselektion und intensiven Monitorings, bedeutend niedriger; sie korrelieren eindeutig mit der kumulativen MTX-Dosis. Bemerkenswerterweise haben Patienten, die MTX aufgrund einer Psoriasis oder Psoriasisarthritis erhalten, bei gleicher kumulativer Dosis ein fast dreifach höheres Risiko, eine Lebertoxizität zu entwickeln als Patienten, die dieses Medikament zur Therapie einer rheumatoiden Arthritis erhalten. Als Erklärung hierfür wird u. a. ein erhöhter Alkoholkonsum bei Psoriasispatienten diskutiert; letztlich ist die Ursache jedoch unklar. Untersuchungen an Patienten mit rheumatoider Arthritis haben gezeigt, dass die zusätzliche Gabe von Folsäure die Inzidenz von Leberfunktionsstörungen senkt, ohne die Wirksamkeit von MTX zu reduzieren. Daher wird heute gemeinhin eine Gabe von $5 \mathrm{mg}$ Folsäure am Tag nach der MTX-Einnahme empfohlen.

Eine beginnende oder manifeste Leberfibrose oder -zirrhose spiegelt sich nicht immer in einer laborchemischen Erhöhung der Leberwerte wider, so dass Routine-Laborkontrollen allein zum Erkennen solcher Toxizitäten nicht ausreichend sind. Nach den Empfehlungen der American Academy of Dermatology sollten unter MTX-Therpie in regelmäßigen Abständen Leberbiopsien erfolgen, abhängig von weiteren Risikofaktoren jeweils spätestens nach einer kumulativen Dosis von 1,5 g [23]. Die Entscheidung über eine Therapiefortsetzung richtet sich dann jeweils nach dem histologischen Befund. Diese Empfehlungen stehen im Widerspruch zu Leitlinien des American College of Rheumatology, die nicht zu routinemäßigen Leberbiopsien raten. In Europa werden routinemäßige Leberbiopsien, die ja als invasive Eingriffe nicht nur belastend für die Patienten sind, sondern auch selbst mit zahlreichen Komplikationen behaftet sein können, zunehmend in Frage gestellt. In jüngerer Zeit wurde Serum-Typ IIIProkollagen-Aminopeptid (PIINP) als laborchemischer Marker für die Früherkennung einer Leberfibrose etabliert, der nach der aktuellen Studienlage eine hohe Sensitivität besitzt. Demnach haben Patienten mit konsistent normalen PIIINP-Werten ein extrem geringes Risiko, eine Leberfibrose oder -zirrhose zu entwickeln $[28,29]$, so dass Leberbiopsien nur bei wiederholt erhöhten PIIINP-Werten bzw. bei ausgewählten Risikopatienten empfohlen werden [29]. Allerdings besitzt der PIIINP-Wert bei aktiver Psoriasisarthritis nur eine eingeschränkte Aussagekraft [28], und zur definitiven Festlegung seines Stellenwerts bedarf es weiterer Studien.
Eine andere bedeutsame Nebenwirkung von MTX ist seine Hämatotoxizität mit Anämie, Leukopenie, Thrombopenie und Makrozytose, die regelmäßige Kontrollen des Differenzialblutbilds - anfangs in zweiwöchentlichen Abständen, später monatlich erforderlich macht (Tab. 2). Sollten schwerwiegende hämatologische Komplikationen auftreten oder eine akzidentelle Überdosierung erfolgt sein, so kann ein Rescue mit Folsäure (Leukovorin) durchgeführt werden, das alle $6 \mathrm{~h}$ in einer Dosierung von $20 \mathrm{mg}$ appliziert wird. Die als MTX-Nebenwirkung bekannte ulcerative Stomatitis sollte als Warnzeichen hierfür gewertet werden.

Eine seltene, aber potenziell bedrohliche und irreversible Nebenwirkung ist eine durch MTX induzierte fibosierende Pneumonitis. Daher sollten alle Patienten regelmäßig nach Symptomen wie trockenem Husten und Dyspnoe befragt und bei entsprechenden Symptomen sorgfältig untersucht werden.

Häufig und meist eher harmlos sind gastrointestinale Beschwerden wie Übelkeit und Erbrechen. Diese treten besonders nach oraler Gabe von MTX auf. Oft kann hier eine Umstellung auf eine parentale Applikation (intravenös oder intramuskulär) Abhilfe schaffen.

In der Schwangerschaft ist MTX streng kontraindiziert, da es sowohl Aborte hervorrufen als auch teratogen wirken kann. Nach Therapieende sollten sowohl Frauen als auch Männer für drei weitere Monate verhüten.

Sorgfältig geachtet werden muss auf Wechselwirkungen zwischen MTX und anderen Medikamenten [23]. So können insbesondere Trimethoprim/Sulfamethoxazol und Dapson, aber auch nichtsteroidale Antiphlogistika den MTX-Spiegel erhöhen und sollten daher nicht gemeinsam mit MTX gegeben werden.

\section{Zusammenfassende Beurteilung}

MTX ist ein effektives Therapeutikum zur Behandlung der Psoriasis wie auch der Psoriasisarthritis und stellt neben Ciclosporin das am stärksten wirksame klassische systemische Antipsoriatikum dar. Dem gegenüber stehen eine Reihe schwerwiegender Nebenwirkungen und die Notwendigkeit einer intensiven Therapieüberwachung, so dass für jeden Patienten eine sorgfältige Risiko-Nutzen-Abwägung vorgenommen werden sollte. Bei Ausschluss von Risikopatienten und unter engmaschigem Monitoring ist die Therapie jedoch auch über einen längeren Zeitraum recht sicher und gut verträglich [30].

\section{Ciclosporin}

1979 wurde erstmals zufällig bei einem Patienten, der nach einer Nierentransplantation Ciclosporin erhielt, eine Besserung seiner Psoriasis beobachtet [31]. In den 1980er Jahren gewann dieses Medikament zunehmende Popularität, und seine Wirksamkeit für die Psoriasis wurde durch kleinere Studien untermauert; seit 1983 ist es in einigen europäischen Ländern einschließlich Deutschland zur Psoriasistherapie zugelassen. Ciclosporin ist ein potentes Immunsuppressivum, das sowohl zur Therapie der schweren chronisch-stationären Psoriasis vulgaris als auch für akute Psoriasisformen und für die Psoriasisarthopathie einge- 
setzt werden kann. Die empfohlene Dosis liegt zwischen 2,5 und $5 \mathrm{mg} / \mathrm{kg} \mathrm{KG} /$ Tag, verteilt auf zwei Einzeldosen [32]. Ciclosporin existiert in zwei Formulierungen, Sandimmun ${ }^{\circledR}$ und Sandimmun ${ }^{\circledR}$ Optoral, letztere eine Mikroemulsion mit dem Vorteil einer höheren und zuverlässigeren Resorption. Zwischen beiden Formulierungen kann die Dosis im Verhältnis 1:1 konvertiert werden.

\section{Wirkmechanismus}

Ciclosporin wirkt immunsuppressiv, indem es die erste Phase der T-Zell-Aktivierung inhibiert: Es bildet zunächst einen Komplex mit dem Immunophilin Cyclophilin, welcher dann an das Enzym Calcineurin, eine Kalzium-aktivierte Phosphatase bindet und diese blockiert. Hierdurch werden Signaltransduktionskaskaden inhibiert, die von dem Transkriptionsfaktor NF-AT (nuclear factor of activated $T$ cells) abhängen, was letztendlich die Produktion bestimmter Zytokine wie Interleukin-2 und Interferon- $\gamma$ unterdrückt. Darüber hinaus inhibiert Ciclosporin die Antigenpräsentation durch dendritische Zellen und die Expression zellulärer Adhäsionsmoleküle wie E-Selektin und ICAM-1.

\section{Effektivität}

Die Wirksamkeit von Ciclosporin in der Psoriasistherapie ist in einer Reihe von Studien gut belegt: In einer systematischen Literaturübersicht wurden 18 randomisierte Studien identifiziert [13], deren Ergebnisse zwar aufgrund unterschiedlicher Dosierungsschemata und Therapiezeiten recht heterogen sind, aus denen sich aber zusammenfassend ableiten lässt, dass CiclosporinDosierungen zwischen 2,5 und $5 \mathrm{mg} / \mathrm{kg} \mathrm{KG} / \mathrm{Tag}$ sehr effizient und rasch Remissionen induzieren können. Die erste doppelblinde, Plazebo-konrollierte Studie zur Dosisfindung wurde 1991 von Ellis und Kollegen durchgeführt und zeigte nach Gabe von Plazebo, $3 \mathrm{mg}, 5 \mathrm{mg}$ und 7,5 mg/kg KG Ciclosporin PASI-Verbesserungen um $0 \%, 39 \%, 58 \%$ und $71 \%$ [34]. Darüber hinaus konnte in mehreren Studien belegt werden, dass mit Dosierungen um $3 \mathrm{mg} / \mathrm{kg} \mathrm{KG}$ die Aufrechterhaltung einer einmal vorhandenen Remission möglich ist [13]. So zeigte z. B. eine Studie von Shupack et al. nach 16 Wochen einer Initialtherapie mit $5 \mathrm{mg} / \mathrm{kg} \mathrm{KG} \mathrm{Ciclo-}$ sporin eine Reduktion des PASI-Scores um durchschnittlich $86 \%$ und demonstrierte anschließend die Wirksamkeit einer Erhaltungstherapie mit $3 \mathrm{mg} / \mathrm{kg}$ KG Ciclosporin: Während in der Patientengruppe, die Plazebo erhielt, 84\% der Patienten innerhalb von 24 Wochen ein Rezidiv erlitten, geschah dies in der mit Ciclosporin behandelten Gruppe nur bei $42 \%$ der Patienten [35]. Eine große Studie an 521 Patienten, die mit Ciclosporin-Dosierungen von 2,5 mg versus $5 \mathrm{mg} / \mathrm{kg}$ KG über 21 Monate behandelt wurden, ergab bei $52 \%$ respektive $92 \%$ der Patienten einen PASI-75 [36]. Ciclosporin ist auch bei pustulösen und lokalisierten pustulösen Formen der Psoriasis wirksam.

\section{Sicherheitsprofil}

Die wichtigsten mit Ciclosporin assoziierten Nebenwirkungen sind Nephrotoxizität, Erhöhung des Blutdrucks und Immunsuppression (Tab.1). Die Nephrotoxizität ist dosisabhängig und tritt bei langzeitiger Gabe höherer Dosen fast unvermeidlich ein [33]; sie kann akut oder chronisch sein. Akute Nephrotoxizität wird durch eine Vasokonstriktion der Arteriolae afferentes und, damit einhergehend, durch eine Reduktion der glomerulären Filtrationsrate induziert. Chronische Nephrotoxizität mit einer Erhöhung des Serumkreatinins wird nach retrospektiven Studien bei
24-46\% der Patienten beobachtet [22], ist jedoch nach Dosisreduktion oder nach Absetzen meist reversibel. Seltener - und abhängig von der Therapiedauer und Dosis - können irreversible strukturelle Veränderungen der Nierentubuli der Arteriolen auftreten. Aufgrund dieses Risikos ist ein sorgfältiges Monitoring der Patienten erforderlich: Sollte der Kreatininwert im Therapieverlauf den Ausgangswert um mehr als 25\% überschreiten, ist eine Dosisreduktion erforderlich. Darüber hinaus wird unter $\mathrm{Ci}$ closporin eine halbjährliche Bestimmung der Kreatininclearance empfohlen [22].

Ein weiteres Risiko ist die Induktion einer arteriellen Hypertonie, die bei bis zu 1/4 der Patienten auftritt und sich meist schleichend über Monate entwickelt. Falls unter Ciclosporin ein Blutdruckanstieg auffällt, sollte die Dosis reduziert werden. Darüber hinaus kann aber auch eine medikamentöse antihypertensive Therapie erforderlich sein; Kalziumkanalantagonisten gelten hierfür als Mittel der ersten Wahl.

Als Immunsuppressivum erhöht Ciclosporin das Infektionsrisiko und ist außerdem mit einer erhöhten Tumorinzidenz verbunden. Das vermehrte Auftreten von Malignomen v. a. lymphoproliferativen Ursprungs während oder nach einer Ciclosporin-Therapie ist für Transplantationspatienten gut belegt, und auch für Psoriasispatienten liegen hierfür Hinweise vor, trotz einer meist kürzeren Therapiedauer und niedrigeren Dosis. In einer prospektiv über fünf Jahre durchgeführten Studie an 1252 Patienten, die Ciclosporin über durchschnittlich 1,9 Jahre erhalten hatten, entwickelten 3,8\% ein Malignom [37]. Dies entspricht einer im Vergleich zur Allgemeinbevölkerung verdoppelten Inzidenz und lässt sich v. a. auf das gehäufte Auftreten von Spinaliomen zurückführen. deren Inzidenz sechsfach erhöht war. Spinaliome wurden v. a. bei solchen Patienten beobachtet, die früher eine PUVA-Therapie und/oder eine Therapie mit anderen Immunsuppressiva erhalten hatten; solche Patienten sollten daher regelmäßig - möglichst halbjährlich - auf Hauttumoren kontrolliert werden.

Bekannte Nebenwirkungen von Ciclosporin, die ungefährlich, aber für die Patienten sehr belastend sein können, sind Tremor, gingivale Hyperplasie und Hypertrichose; für weitere unerwünschte Wirkungen sei auf Tab. 1 verwiesen.

Ciclosporin ist das einzige systemische Antipsoriatikum, für das in der Schwangerschaft keine absolute Kontraindikation besteht. Hier ist aber selbstverständlich eine besonders sorgfältige Kosten-Nutzen-Abwägung angezeigt.

\section{Zusammenfassende Beurteilung}

Ciclosporin kann eine zügige Besserung, manchmal auch eine Abheilung psoriatischer Hautläsionen bewirken und hat daher einen besonderen Stellenwert in der Induktionstherapie schwerer Psoriasis-Formen. Für eine kurzfristige Behandlung wird dabei das Nutzen-Risiko-Verhältnis als akzeptabel angesehen. Aufgrund der eindeutigen Beziehung zwischen der kumulativen Ciclosporin-Dosis und schwerwiegenden Toxizitäten verbietet sich jedoch eine kontinuierliche Therapie für länger als 1-2 Jahre $[22,30,33]$. Vorzuziehen sind kurze Zyklen von 12 Wochen. Durch sorgfältiges Monitoring und, soweit erforderlich, frühzeitige Dosisreduktion können Toxizitäten oft minimiert werden. 
Bei Patienten, deren Lebensqualität durch die Schwere der Psoriasis erheblich eingeschränkt ist, wird man sich eher zugunsten von Ciclosporin entscheiden, ebenso bei Patienten, bei denen andere Therapieoptionen wie Acitretin und MTX aufgrund von Kontraindikationen entfallen. Beispiele hierfür können Frauen im gebärfähigen Alter sein, die keine absolut zuverlässige Kontrazeption gewährleisten können oder wollen. Demgegenüber wird die Nutzen-Risiko-Abwägung für ältere Patienten mit einem höheren Risiko für eine Nierenfunktionsstörung und eine Hypertonie weniger günstig ausfallen, gleichermaßen für Patienten mit längerfristiger immunsuppressiver Therapie in der Vorgeschichte.

\section{Leflunomid}

Leflunomid ist ein Pyrimidinantagonist, der in der Therapie der rheumatoiden Arthritis seinen festen Platz hat [38]. Zur Therapie der Psoriasisarthritis ist Leflunomid in Deutschland seit Juni 2004 zugelassen, für die Psoriasis vulgaris ohne Gelenkbeteiligung besteht keine Zulassung. Zur Aufsättigung wird Leflunomid zunächst über drei Tage in einer Dosierung von $100 \mathrm{mg}$ verabreicht. Die Erhaltungsdosis beträgt danach $20 \mathrm{mg} /$ Tag. Ein Wirkeintritt ist nach vier bis sechs Wochen zu erwarten.

\section{Wirkmechanismus}

Leflunomid ist eine Prodrug, deren M1-Metabolit A771726 die Dihydroorotat-Dehydrogenase, ein Schlüsselenzym in der Pyrimidinsynthese, blockiert. Dadurch wird der Zellzyklus in der G1-Phase arretiert und die Proliferation v. a. von Lymphozyten inhibiert. Dies führt letztlich zu einer verminderten Synthese proinflammatorischer Zytokine.

\section{Effektivität}

Während die Wirksamkeit von Leflunomid für die Therapie der rheumatoiden Arthritis gut belegt ist [38], existiert für die Indikation Psoriasisarthritis bisher nur eine größere Studie, die an insgesamt 190 Patienten doppelblind und Plazebo-kontrolliert durchgeführt wurde [39]. Die Ergebnisse weisen auf ein recht gutes Ansprechen der Arthritis bei weniger gutem Ansprechen der Hautläsionen hin. So konnte innerhalb von 24 Wochen bei $59 \%$ der mit Leflunomid behandelten Patienten gegenüber 30\% der Patienten in der Plazebo-Gruppe eine Besserung der Arthritis dokumentiert werden. Demgegenüber wurde eine 50\%ige Besserung des PASI nur bei 30\% der Patienten mit Leflunomid-Therapie versus $19 \%$ der Patienten in der Plazebogruppe erreicht. Ein PASI-75 ließ sich sogar nur in 17 versus $8 \%$ der Fälle erzielen. Diese Befunde wurden im Wesentlichen durch kleinere Studien und Fallberichte untermauert.

\section{Sicherheitsbedenken}

Eine recht häufige unerwünschte Wirkung von Leflunomid betrifft Leberwert-Erhöhungen (Tab.1). Diese sind meist nach Dosisreduktion oder Absetzen des Medikaments reversibel; in seltenen Fällen wurden jedoch schwere Leberschäden berichtet, vereinzelt auch mit tödlichem Ausgang. Aus diesem Gründen ist Leflunomid bei vorbestehender Lebererkrankung kontraindiziert. Unter Therapie sollten zusätzliche hepatotoxische Medikamente und der Konsum von Alkohol möglichst vermieden werden, ebenso sind die Leberfunktionsparameter engmaschig zu kontrollie- ren (Tab. 2). Bei einem Anstieg der ALAT auf das Zweifache der Norm sollte die Dosis halbiert werden, bei einem Anstieg über das Dreifache ist ein Abbruch der Therapie angezeigt [40].

Weitere Toxizitäten, auf die geachtet werden muss, sind Blutbildveränderungen sowie ein Anstieg des Blutdrucks. Zusätzlich können relativ häufig gastrointestinale Beschwerden wie Übelkeit und Durchfall, Haarausfall und allergische Reaktionen auftreten. Seltene Nebenwirkungen sind periphere Neuropathien, schwere allergische Reaktionen und interstitielle Pneumonien. In klinischen Studien mit der Indikation rheumatoide Arthritis brachen 14-22\% der Patienten die Leflunomid-Therapie aufgrund von Nebenwirkungen ab. Diese Zahlen liegen im klinischen Alltag allerdings sogar um $30 \%$.

Der aktive Metabolit von Leflunomid, A771726, ist teratogen und besitzt darüber hinaus eine Eliminationshalbwertszeit von bis zu vier Wochen. Daher ist Lefluonmid bei Frauen im gebärfähigen Alter kontraindiziert, es sei denn, dass unter Therapie und zwei Jahre danach eine zuverlässige Kontrazeption gewährleistet ist. Wird nach Therapieende eine Schwangerschaft gewünscht, muss nach der vorgeschriebenen Wartezeit von zwei Jahren der Plasmaspiegel von A771726 bestimmt werden. Dieser muss zweimal im Abstand von 14 Tagen unter 0,02 mg/l liegen, damit ein teratogenes Risiko mit hoher Wahrscheinlichkeit ausgeschlossen ist. Auch für Männer ist eine Kontrazeption unter Therapie erforderlich; die Wartezeit nach Therapieende beträgt hier drei Monate. Um eine schnellere Elimination von Leflunomid zu erreichen, können Cholestyramin oder alternativ Aktivkohle verabreicht werden [40].

\section{Zusammenfassende Beurteilung}

Leflunomid kommt zur Therapie der Psoriasisarthritis am ehesten bei solchen Patienten in Frage, bei denen der Hautbefall gering ausgeprägt ist und an sich keine systemische Therapie erfordern würde. Für Patienten mit aktiver Psoriasisarthritis, die gleichzeitig ausgeprägte psoriatische Hautläsionen aufweisen, erscheint Leflunomid weniger geeignet; hier kämen nach einem erfolglosen Versuch mit MTX eher TNF $\alpha$-Antagonisten wie Etanercept in Frage. Faktoren, die den Einsatz von Leflunomid limitieren können, sind u. a. die Hepatotoxizität, gastrointestinale Beschwerden sowie allergische Reaktionen und das teratogene Risiko.

\section{Mycophenolat-Mofetil}

Mycophenolat-Mofetil (MMF) ist ein Immunsuppressivum, das zur Prophylaxe und Therapie von Abstoßungsreaktionen in der Transplantationsmedizin eingesetzt wird und in jüngerer Zeit zunehmend für Hautkrankheiten, in erster Linie für Autoimmundermatosen, entdeckt wurde. Erste Berichte über den Einsatz seines aktiven Metaboliten, Mycophenolsäure, zur Behandlung der Psoriasis stammen aus den 1970er Jahren. Seither wurden mehrere kleinere Studien durchgeführt; eine Zulassung für die Psoriasistherapie liegt bislang allerdings noch nicht vor. Zur Therapie von Autoimmunerkrankungen der Haut wird MMF in einer Dosis von maximal $2 \mathrm{~g} / \mathrm{Tag}$, verteilt auf zwei bis vier Einzelgaben, eingesetzt; für die Psoriasistherapie wurden ähnliche Dosierungen verwendet. 


\section{Wirkmechanismus}

MMF inhibiert die Purinsynthese von Lymphocyten, indem es reversibel das Enzym Inosinmonophosphat-Dehydrogenase blockiert und die Synthese von Guanosinmonophosphat verhindert. Andere Zellen können Guanosinmonophosphat alternativ auch über den Hypoxanthin-Guanin-Phosphoribosyltransferase-Weg produzieren; da Lymphozyten diese Fähigkeit nicht besitzen, blockiert MMF ihre Proliferation selektiv.

\section{Effektivität, Sicherheitsprofil und Perspektiven}

Zum Einsatz von MMF in der Psoriasistherapie liegen bisher keine randomisierten Studien und keine Studien an größeren Patientenkollektiven vor. In einer kleinen offenen Studie an $11 \mathrm{~Pa}-$ tienten konnten Geilen und Kollegen mit einer Dosis von $2 \mathrm{~g}$ MMF/Tag innerhalb von drei Wochen bei 7 von 11 Patienten eine PASI-Reduktion zwischen 40 und 70\% erreichen [41]. Die anschließend gewählte Dosis von $1 \mathrm{~g} /$ Tag war weniger effektiv. Eine weitere, an 23 Patienten durchgeführte offene Studie mit 2-3 g MMF ergab nach 6 Wochen eine 24\%ige und nach 12 Wochen eine $47 \%$ ige Reduktion des PASI-Scores, wobei insgesamt $77 \%$ der Patienten auf die Therapie ansprachen [42]. In einer Fallserie zur Psoriasisarthritis zeigte MMF in 4 von 6 Patienten einen günstigen Effekt [43].

Eine abschließende Beurteilung der Effektivität von MMF in der Psoriasistherapie ist derzeit bei Fehlen größerer randomisierter Studien nicht möglich. Die bisherige Datenlage weist jedoch auf eine insgesamt eher mittelmäßige Wirksamkeit hin. So kam es z. B. nach Umstellung von Ciclosporin auf MMF bei 5 von 8 Patienten zu einer deutlichen Exazerbation der Psoriasis [44].

\section{Sicherheitsprofil}

Im Vergleich mit anderen Immunsuppressiva besitzt MMF ein recht günstiges Sicherheitsprofil. Die am häufigsten beobachteten unerwünschten Wirkungen sind gastrointestinale Beschwerden, die sich jedoch oft durch eine Verteilung der Tagesdosis auf 2-4 Einzeldosen lindern lassen. Darüber hinaus muss auf eine Myelosuppression und auf eine erhöhte Infektanfälligkeit, auch für opportunistische Infektionen, geachtet werden. Wie bei allen Immunsuppressiva ist v. a. nach jahrelanger Gabe mit einem erhöhten Risiko für die Entwicklung von Tumoren zu rechnen.

\section{Zusammenfassende Beurteilung}

Zur Bestimmung des Stellenwerts von MMF in der Psoriasistherapie sind kontrollierte Studien erforderlich. Die bisherige Datenlage deutet auf eine im Vergleich mit Ciclosporin geringere Wirksamkeit hin, allerdings mit dem Vorteil einer recht guten Verträglichkeit und eines insgesamt eher günstigeren Sicherheitsprofils. Da MMF bisher nicht zur Psoriasistherapie zugelassen ist, muss ein Therapieversuch außerhalb von Studien als individueller Heilversuch erfolgen. Ein solcher kann in Einzelfällen, z. B. bei Kontraindikationen gegen mehrere andere zugelassene Therapieformen, einschließlich der Biologicals, sinnvoll sein.
Kombinationsmöglichkeiten verschiedener systemischer Therapien

Falls eine systemische Monotherapie allein nicht zu einer ausreichenden Verbesserung der Schuppenflechte führt, sind Kombinationstherapien möglich (Übersicht: z. B. [45]). Diese bieten den Vorteil, dass durch unterschiedliche Wirkmechanismen synergistische Effekte erzielt werden können. Beispielsweise wirken manche Systemtherapeutika hauptsächlich auf die epidermale Differenzierung, während andere in erster Linie immunsuppressiv und anti-inflammatorisch sind. Dadurch können die einzelnen Medikamente oft niedriger dosiert werden, was letztendlich das Toxizitätsrisiko reduziert. Typischerweise wird bei einer Kombinationstherapie zunächst mit beiden Medikamenten eine Remission induziert. Sobald diese erreicht ist, wird eines der beiden - vorzugsweise das nebenwirkungsreichere - abgesetzt; das andere wird über einen gewissen Zeitraum als Erhaltungstherapie fortgeführt.

Am häufigsten wird eine Kombination aus Acitretin mit einer Lichttherapie eingesetzt (s. o.). Nachdem mit Hilfe einer solchen Kombination eine wesentliche Verbesserung oder Abheilung erreicht ist, sind verschiedene Vorgehensweisen möglich: Einerseits kann die Lichttherapie ausgeschlichen und Acitretin niedrig dosiert als Erhaltungstherapie fortgesetzt werden. Eine andere Alternative wäre, Acitretin abzusetzen und die Lichttherapie für einen gewissen Zeitraum zur Stabilisierung des Hautbefunds fortzusetzen. Acitretin wurde bei Risikopatienten wie z. B. Transplantationspatienten erfolgreich zur Chemoprophylaxe von epithelialen Hauttumoren eingesetzt [21] und ist auch zur Prävention lichtinduzierter Hauttumore bei Psoriasispatienten wirkungsvoll.

Acitretin kann auch mit Ciclosporin kombiniert werden [16, 22]: Nach einem von Koo vorgeschlagenen Schema wird dabei zunächst durch Einsatz von Ciclosporin eine Remission induziert [16]. Wenn diese erzielt ist, wird in einer zweiten Phase schrittweise Acitretin hinzugegeben und die höchstmögliche gut verträgliche Dosis ermittelt. Hierbei muss besonders auf die Blutfettwerte geachtet werden, da diese durch beide Medikamente erhöht werden können. Anschließend wird Ciclosporin schrittweise ausgeschlichen.

Für ausgeprägte und extrem therapierefraktäre Falle einer Psoriasis werden Kombinationen von Ciclosporin und MTX empfohlen, wobei beides eher niedrig dosiert werden sollte und ein engmaschiges Monitoring angezeigt ist. In einer retrospektiven Studie mit 19 Patienten wurde die Kombination insgesamt gut vertragen und ermöglichte eine gute Kontrolle des Hautbefunds und der Arthropathie [46]. Auch bei schwerer Psoriasisarthritis kann eine solche Kombination erwogen werden, wenn mit MTX allein nur eine partielle Besserung erzielt wurde.

Darüber hinaus wurden in jüngerer Zeit zunehmend Kombinationen von klassischen Systemtherapien mit Biologicals eingesetzt. Zur Therapie der rheumatoiden Arthritis ist MTX zusammen mit dem TNFo-Antagonisten Etanercept schon seit Jahren zugelassen, und für diese Indikation liegen bereits ausgedehnte Erfahrungen und größere randomisierte Studien vor [47]. Auch für die Psoriasisarthritis scheinen MTX plus Etanercept gegen- 
über der jeweiligen Monotherapie überlegen zu sein [48]. Während diese Kombination in den USA bereits für die Therapie der Psoriasisarthritis zugelassen ist, steht eine solche Zulassung in Deutschland noch aus.

Zur Therapie der rheumatoiden Arthritis kann MTX auch mit Infliximab kombiniert werden [49]. Hierbei wurde allerdings gegenüber einer MTX-Monotherapie eine deutlich erhöhte Infektionsrate berichtet; überwiegend traten Infektionen des oberen Respirationstrakts, Pharyngitiden und Sinusitiden auf, aber auch schwere Infektionen wie Sepsis. Für die Indikationen Psoriasis und Psoriasisarthritis liegen für diese Kombination, genau wie für andere Kombinationen mit Biologicals, nur kleinere Fallserien bzw. offene Studien vor: Covelli et al. konnten in einer offenen Studie an 26 Patienten mit MTX plus Infliximab ein deutlich besseres Ansprechen der Psoriasisarthritis als unter einer MTX-Monotherapie belegen. Allerdings kam es nach Absetzen von Infliximab bei den meisten Patienten recht schnell - innerhalb von 2 bis 6 Monaten - zu einem Rezidiv [50]. Ein erhöhtes Risiko für das Auftreten opportunistischer Infektionen besteht auch hier. Des Weiteren muss auf längere Sicht mit einem deutlich erhöhten Malignom-Risiko gerechnet werden; wie hoch dieses Risiko tatsächlich ist, wird die Zukunft erweisen.

Die Kombination einer Ciclosporin- mit einer Lichttherapie ist kontraindiziert, da hierunter ein erhöhtes Risiko für Hauttumoren zu befürchten ist; gleiches gilt für MTX. Kombinationen von Acitretin mit MTX sollten wegen des erhöhten Risikos einer Leberschädigung vermieden werden.

\section{Rotationstherapie und sequentielle Therapie}

Hauptziel einer Rotationstherapie ist es, kumulative Toxizitäten zu vermeiden, indem von einer zur anderen Therapie gewechselt wird. Das erste Beispiel, das hierfür beschrieben wurde, ist eine alternierende Therapie mit UVB plus Teer, PUVA, MTX und Acitretin. Seither hat sich - u. a. mit Einführung der Biologicals das Therapiespektrum wesentlich erweitert, und Biologicals können Teil einer solchen Rotationstherapie sein.

Für sequentielle Therapien werden verschiedene Therapien in einer bestimmten Reihenfolge mit dem Ziel hintereinander geschaltet, mit einem hochwirksamen Medikament möglichst schnell eine Remission zu induzieren, dieses in einer Übergangsphase schrittweise durch ein nebenwirkungsärmeres Medikament zu ersetzen und schließlich in der Erhaltungsphase ganz auf das besser verträgliche Medikament überzugehen. Ein geläufiges Beispiel hierfür ist die weiter oben beschriebene Kombination von Ciclosporin mit Acitretin. Rotationstherapien und sequentielle Therapien sind in der Therapie der Psoriasis insofern unerlässlich, als es sich um eine chronisch-rezidivierende, lebenslang bestehende Erkrankung handelt, die eines langfristigen Behandlungskonzepts bedarf. Wann immer möglich, sollte solchen Therapieansätzen gegenüber einer systemischen Dauertherapie der Vorzug gegeben werden.
Fazit

Die verfügbaren klinischen Daten zeigen, dass klassische systemische Therapien psoriatische Haut- und Gelenkveränderungen effektiv beeinflussen können. Die beiden wirksamsten Medikamente sind MTX und Ciclosporin; ihr langfristiger Einsatz über Jahre und Jahrzehnte wird jedoch durch das Risiko kumulativer Toxizitäten limitiert. Daher bieten sich, wo immer möglich, intermittierende, sequentielle oder Rotationstherapien an.

Das Therapiespektrum der Psoriasis hat sich in den letzen Jahren durch die Biologicals wesentlich erweitert. Ihre z. T. sehr gute Wirksamkeit ist in den vergangenen Jahren gut dokumentiert worden. Die Evaluation ihrer Effektivität und Sicherheit in der langfristigen Therapie der Psoriasis steht allerdings noch aus. Sicherlich werden die Biologicals in Zukunft eine größere Rolle in der Therapie der Psoriasis spielen; ob sie die klassischen Antipsoriatika werden ersetzen können, ist heute noch nicht absehbar.

\section{Literatur}

${ }^{1}$ Rapp SR, Feldman SR, Exum ML, Fleischer AB Jr, Reboussin DM. Psoriasis causes as much disability as other major medical diseases. J Am Acad Dermatol 1999; 41: $401-407$

${ }^{2}$ Feldman SR, Koo JY, Menter A, Bagel J. Decision points for the initiation of systemic treatment for psoriasis. J Am Acad Dermatol 2005; 53: $101-107$

${ }^{3}$ Schweckendieck W. Heilung von Psoriasis. Med Monatsschr 1959; 13: $103-104$

${ }^{4}$ Fumaderm initial/Fumaderm magensaftresistente Tabletten. Fachinformation.

${ }^{5}$ Mrowietz U, Christophers E, Altmeyer P. Treatment of severe psoriasis with fumaric acid esters: scientific background and guidelines for therapeutic use. The German Fumaric Acid Ester Consensus Conference. Br J Dermatol 1999; 141: 424-429

${ }^{6}$ Nugteren-Huying WM, van der Schroeff JG, Hermans J, Suurmond D. Fumaric acid therapy for psoriasis: a randomized, double-blind, placebo-controlled study. J Am Acad Dermatol 1990; 22: 311 - 312

${ }^{7}$ Altmeyer PJ, Matthes U, Pawlak F, Hoffmann K, Frosch PJ, Ruppert P, Wassilew SW, Horn T, Kreysel HW, Lutz G et al. Antipsoriatic effect of fumaric acid derivatives. Results of a multicenter double-blind study in 100 patients. J Am Acad Dermatol 1994; 30: 977-981

${ }^{8}$ Mrowietz U, Christophers E, Altmeyer P. Treatment of psoriasis with fumaric acid esters: results of a prospective multicentre study. German Multicentre Study. Br J Dermatol 1998; 138: 456 - 460

${ }^{9}$ Gollnick H, Altmeyer P, Kaufmann R, Ring J, Christophers E, Pavel S, Ziegler J. Topical calcipotriol plus oral fumaric acid is more effective and faster acting than oral fumaric acid monotherapy in the treatment of severe chronic plaque psoriasis vulgaris. Dermatology 2002; 205: $46-53$

${ }^{10}$ Altmeyer P, Hartwig R, Matthes U. Das Wirkungs- und Sicherheitsprofil von Fumarsäureestern in der oralen Langzeittherapie bei schwerer therapieresistenter Psoriasis vulgaris. Eine Untersuchung an $83 \mathrm{~Pa}-$ tienten. Hautarzt 1996; 47: 190-196

${ }^{11}$ Neotigason. Fachinformation.

${ }^{12}$ Saurat JH. Retinoids and psoriasis: novel issues in retinoid pharmacology and implications for psoriasis treatment. J Am Acad Dermatol 1999; 41: S2 - S6

${ }^{13}$ Griffiths CEM, Clark CM, Chalmers RJG, Li Wan Po A, Williams HC. A systematic review of treatments for severe psoriasis. Health Technology Assessment 2000; 4: 1-115

${ }^{14}$ Anadkat MJ, Heffernan MR. Systemic retinoids. In: Gordon KB, Ruderman EM (eds). Psoriasis and psoriatic arthritis. Berlin, Heidelberg, New York: Springer, 2005: $158-172$

${ }^{15}$ Mahrle G, Schulze HJ, Farber L, Weidinger G, Steigleder GK. Low-dose short-term cyclosporine versus etretinate in psoriasis: improvement 
of skin, nail, and joint involvement. J Am Acad Dermatol 1995; 32 : 78 88

${ }^{16}$ Koo J. Systemic sequential therapy of psoriasis: a new paradigm for improved therapeutic results. J Am Acad Dermatol 1999; 41: S25-S28

17 Tanew A, Guggenbichler A, Honigsmann H, Geiger JM, Fritsch P. Photochemotherapy for severe psoriasis without or in combination with acitretin: a randomized, double-blind comparison study. J Am Acad Dermatol 1991; 25: $682-684$

${ }^{18}$ Ruzicka T, Sommerburg C, Braun-Falco O, Koster W, Lengen W, Lensing W, Letzel H, Meigel WN, Paul E, Przybilla B et al. Efficiency of acitretin in combination with UV-B in the treatment of severe psoriasis. Arch Dermatol 1990; 126: 482-486

${ }^{19}$ Spuls PI, Rozenblit M, Lebwohl M. Retrospective study of the efficacy of narrowband UVB and acitretin. J Dermatolog Treat 2003; 14: 17-20

${ }^{20}$ Van de Kerkhof PC, Cambazard F, Hutchinson PE, Haneke E, Wong E, Souteyrand P, Damstra RJ, Combemale P, Neumann MH, Chalmers RJ, Olsen L, Revuz J. The effect of addition of calcipotriol ointment (50 micrograms/g) to acitretin therapy in psoriasis. Br J Dermatol 1998; 138: $84-89$

${ }^{21}$ Bavinck JN, Tieben LM, Van der Woude FJ, Tegzess AM, Hermans J, ter Schegget J, Vermeer BJ. Prevention of skin cancer and reduction of keratotic skin lesions during acitretin therapy in renal transplant recipients: a double-blind, placebo-controlled study. J Clin Oncol 1995; 13: $1933-1938$

22 Cather JC, Menter A. Methotrexate and cyclosporin. In: Gordon KB, Ruderman EM (eds). Psoriasis and psoriatic arthritis. Berlin Heidelberg New York: Springer, 2005: $172-184$

${ }^{23}$ Roenigk HH Jr, Auerbach R, Maibach H, Weinstein G, Lebwohl M. Methotrexate in psoriasis: consensus conference. J Am Acad Dermatol 1998; $38: 478-485$

${ }^{24}$ Roenigk HH Jr, Fowler-Bergfeld W, Curtis GH. Methotrexate for psoriasis in weekly oral doses. Arch Dermatol 1969; 99: 86-93

${ }^{25}$ Heydendael VM, Spuls PI, Opmeer BC, de Borgie CA, Reitsma JB, Goldschmidt WF, Bossuyt PM, Bos JD, de Rie MA. Methotrexate versus cyclosporine in moderate-to-severe chronic plaque psoriasis. N Engl J Med 2003; 349: 658-665

${ }^{26}$ Sandhu K, Kaur I, Kumar B, Saraswat A. Efficacy and safety of cyclosporine versus methotrexate in severe psoriasis: a study from north India. J Dermatol 2003; 30: 458 - 463

27 Zachariae H. Have methotrexate-induced liver fibrosis and cirrhosis become rare? A matter for reappraisal of routine liver biopsies. Dermatology 2005; 211: $307-308$

${ }^{28}$ Zachariae H, Heickendorff L, Sogaard $\mathrm{H}$. The value of amino-terminal propeptide of type III procollagen in routine screening for methotrexate-induced liver fibrosis: a 10-year follow-up. Br J Dermatol 2001; 144: $100-103$

${ }^{29}$ Chalmers RJ, Kirby B, Smith A, Burrows P, Little R, Horan M, Hextall JM, Smith $\mathrm{CH}$, Klaber M, Rogers S. Replacement of routine liver biopsy by procollagen III aminopeptide for monitoring patients with psoriasis receiving long-term methotrexate: a multicentre audit and health economic analysis. Br J Dermatol 2005; 152: 444-450

${ }^{30}$ Naldi L, Griffiths CE. Traditional therapies in the management of moderate to severe chronic plaque psoriasis: an assessment of the benefits and risks. Br J Dermatol 2005; 152: 597-615

${ }^{31}$ Mueller W, Herrmann B. Cyclosporin A for psoriasis. N Engl J Med 1979; 301: 555

32 Sandimmun optoral. Fachinformation.

${ }^{33}$ Griffiths CE, Dubertret L, Ellis CN, Finlay AY, Finzi AF, Ho VC, Johnston A, Katsambas A, Lison AE, Naeyaert JM, Nakagawa H, Paul C, Vanaclocha F. Cyclosporin in psoriasis clinical practice: an international consensus statement. Br J Dermatol 2004; 150 (Suppl 67): 11 - 23

${ }^{34}$ Ellis CN, Fradin MS, Messana JM, Brown MD, Siegel MT, Hartley AH, Rocher LL, Wheeler S, Hamilton TA, Parish TG et al. Cyclosporine for plaque-type psoriasis. Results of a multidose, double-blind trial. N Engl J Med 1991; 324: 277-284
${ }^{35}$ Shupack J, Abel E, Bauer E, Brown M, Drake L, Freinkel R, Guzzo C, Koo J, Levine N, Lowe N, McDonald C, Margolis D, Stiller M, Wintroub B, Bainbridge C, Evans S, Hilss S, Mietlowski W, Winslow C, Birnbaum JE. Cyclosporine as maintenance therapy in patients with severe psoriasis. J Am Acad Dermatol 1997; 36: 423 -432

${ }^{36}$ Laburte C, Grossman R, Abi-Rached J, Abeywickrama KH, Dubertret L. Efficacy and safety of oral cyclosporin A (CyA; Sandimmun) for longterm treatment of chronic severe plaque psoriasis. Br J Dermatol 1994; 130: $366-375$

37 Paul CF, Ho VC, McGeown C, Christophers E, Schmidtmann B, Guillaume JC, Lamarque V, Dubertret L. Risk of malignancies in psoriasis patients treated with cyclosporine: a 5 y cohort study. J Invest Dermatol 2003; 120: 211 - 216

${ }^{38}$ Maddison P, Kiely P, Kirkham B, Lawson T, Moots R, Proudfoot D, Reece R, Scott D, Sword R, Taggart A, Thwaites C, Williams E. Leflunomide in rheumatoid arthritis: recommendations through a process of consensus. Rheumatology (Oxford) 2005; 44: 280-286

${ }^{39}$ Kaltwasser JP, Nash P, Gladman D, Rosen CF, Behrens F, Jones P, Wollenhaupt J, Falk FG, Mease P. Treatment of Psoriatic Arthritis Study Group. Efficacy and safety of leflunomide in the treatment of psoriatic arthritis and psoriasis: a multinational, double-blind, randomized, placebo-controlled clinical trial. Arthritis Rheum 2004; 50: 19391950

40 Arava. Fachinformation.

${ }^{41}$ Geilen CC, Arnold M, Orfanos CE. Mycophenolate mofetil as a systemic antipsoriatic agent: positive experience in 11 patients. $\mathrm{Br} \mathrm{J}$ Dermatol 2001; 144: $583-586$

42 Zhou Y, Rosenthal D, Dutz J, Ho V. Mycophenolate mofetil (CellCept) for psoriasis: a two-center, prospective, open-label clinical trial. J Cutan Med Surg 2003; 7: $193-197$

${ }^{43}$ Schräder P, Mooser G, Peter RU, Puhl W. Erste Ergebnisse der Therapie der Psoriasisarthritis mit Mycophenolatmofetil. Z Rheumatol 2002; 61: $545-550$

${ }^{44}$ Davison SC, Morris-Jones R, Powles AV, Fry L. Change of treatment from cyclosporin to mycophenolate mofetil in severe psoriasis. $\mathrm{Br} \mathrm{J}$ Dermatol 2000; 143: 405-407

${ }^{45}$ Lebwohl M. A clinician's paradigm in the treatment of psoriasis. J Am Acad Dermatol 2005; 53: S59-S69

${ }^{46}$ Clark CM, Kirby B, Morris AD, Davison S, Zaki I, Emerson R, Saihan EM, Chalmers RJ, Barker JN, Allen BR, Griffiths CE. Combination treatment with methotrexate and cyclosporin for severe recalcitrant psoriasis. $\mathrm{Br}$ J Dermatol 1999; 141: 279-282

47 Klareskog L, van der Heijde D, de Jager JP, Gough A, Kalden J, Malaise M, Martin Mola E, Pavelka K, Sany J, Settas L, Wajdula J, Pedersen R, Fatenejad S, Sanda M, TEMPO (Trial of Etanercept and Methotrexate with Radiographic Patient Outcomes) study investigators. Therapeutic effect of the combination of etanercept and methotrexate compared with each treatment alone in patients with rheumatoid arthritis: double-blind randomised controlled trial. Lancet 2004; 363: 675-681

${ }^{48}$ Iyer S, Yamauchi P, Lowe NJ. Etanercept for severe psoriasis and psoriatic arthritis: observations on combination therapy. Br J Dermatol 2002; 146: $118-121$

${ }^{49}$ Lipsky PE, van der Heijde DM, St Clair EW, Furst DE, Breedveld FC, Kalden JR, Smolen JS, Weisman M, Emery P, Feldmann M, Harriman GR, Maini RN, Anti-Tumor Necrosis Factor Trial in Rheumatoid Arthritis with Concomitant Therapy Study Group. Infliximab and methotrexate in the treatment of rheumatoid arthritis. Anti-Tumor Necrosis Factor Trial in Rheumatoid Arthritis with Concomitant Therapy Study Group. N Engl J Med 2000; 343: 1594-1602

${ }^{50}$ Covelli M, Scioscia C, Iannone F, Lapadula G. Repeated infusions of low-dose infliximab plus methotrexate in psoriatic arthritis: immediate benefits are not maintained after discontinuation of infliximab. Clin Exp Rheumatol 2005; 23: 145 - 151 